\title{
ON MULTI-DIMENSIONAL PARTIAL DIFFERENTIAL EQUATIONS WITH POWER NONLINEARITIES IN FIRST DERIVATIVES
}

\section{I.V. RAKHMELEVICH}

\begin{abstract}
We consider a class of multi-dimensional partial differential equations involving a linear differential operator of arbitrary order and a power nonlinearity in the first derivatives. Under some additional assumptions for this operator, we study the solutions of multi-dimensional travelling waves that depend on some linear combinations of the original variables. The original equation is transformed to a reduced one, which can be solved by the separation of variables. Solutions of the reduced equation are found for the cases of additive, multiplicative and combined separation of variables.
\end{abstract}

Keywords: partial differential equation, reduced equation, method of separation of variables, power nonlinearity.

Mathematics Subject Classification: 335G20

\section{INTRODUCTION}

An important direction in the modern mathematical physics is the study of multi-dimensional nonlinear partial differential equations and finding their exact solutions [1-12]. One of the most effective and widely used method of solving such equations is the separation of variables (SV). In known handbooks and textbooks [1-3] the classical scheme of the method is described as well as its modern versions, generalized and functional SV [4]. In works [5-9] by the method of SV, there were studied partial differential equations with power nonlinearities in the derivatives as well as equations involving homogeneous and multi-homogeneous functions of the derivatives; such equations are reduced to the equations with power nonlinearities for certain classes of solutions. The present work is devoted to continuing these studies. We consider a multidimensional partial differential equation involving a linear differential operator of arbitrary order with constant coefficients and power nonlinearities in first derivatives. By means of the reduction method and the method of separation of variables we find the solutions of multidimensional travelling waves type for this equation.

\section{Formulation of the PROBlem}

We consider the following class of multidimensional partial differential equations for an unknown function $u\left(x_{1}, x_{2}, \ldots, x_{N}\right)$ involving power nonlinearities in the first derivatives:

$$
\hat{L} u\left(x_{1}, x_{2}, \ldots, x_{N}\right)=b \prod_{n=1}^{N}\left(\frac{\partial u}{\partial x_{n}}\right)^{\beta_{n}} .
$$

Here $\hat{L}$ is a linear differential operator with constant coefficients in variables $x_{1}, x_{2}, \ldots, x_{N}$.

I.V. RAKHMELEVICH, ON MULTi-DIMENSIONAL PARTIAL DIFFERENTIAL EQUATIONS WiTH POWER NONLINEARITIES IN FIRST DERIVATIVES.

(C) RAKHMELEVICH I.V. 2017.

Submitted October 30, 2015. 
We represent the set of the values $I=\{1, \ldots, N\}$ of the index $n$ indexing independent variables as the union of $K$ disjoint subsets $I_{k},(k=1, \ldots, K)$. Then the set of the variables $X=\left\{x_{1}, x_{2}, \ldots, x_{N}\right\}$ can be partitioned into $K$ disjoint subsets $X_{k}=\left\{x_{n}\right\}_{n \in I_{k}}$. Hereinafter we denote by $\Omega=\{1, \ldots, K\}$ the set of the values of the index $k$. In what follows we assume that the operator $\hat{L}$ can be represented as

$$
\hat{L}=\sum_{k=1}^{K} \hat{L}_{X_{k}}
$$

where $\hat{L}_{X_{k}}$ is a linear differential operator with constant coefficients in the variables $X_{k}$. Relation (2) means that the operator $\hat{L}$ involves no mixed derivatives w.r.t. the variables in different subsets $X_{k}$. In its turn, the operator $\hat{L}_{X_{k}}$ can be represented as a sum of linear homogeneous differential operators of various orders in the variables $X_{k}$ :

$$
\hat{L}_{X_{k}}=\sum_{m=1}^{M_{k}} \hat{L}_{X_{k}}^{(m)}
$$

By the said above, the operator $\hat{L}_{X_{k}}^{(m)}$ can be written as

$$
\hat{L}_{X_{k}}^{(m)}=\sum_{\sigma_{k}^{(m)}} a_{\sigma_{k}^{(m)}} \prod_{n \in I_{k}}\left(\frac{\partial}{\partial x_{n}}\right)^{m_{n}} .
$$

Here we have introduced the multi-index $\sigma_{k}^{(m)}=\left\{m_{n}\right\}_{n \in I_{k}}$, and $m_{n} \geqslant 0$ for all $n \in I_{k}$ and $\sum_{n \in I_{k}} m_{n}=m$. In the present work we seek the solutions to equation (1), which depend on the variables $z_{k}$ and which are linear combinations of the original variables $x_{n}$ :

$$
z_{k}=\sum_{n \in I_{k}} c_{n} x_{n}
$$

In view of relations (2), (3), (4), (5), for the solutions $u=U\left(z_{1}, \ldots, z_{K}\right)$ of the mentioned type, equation (1) can be easily reduced to the form:

$$
\sum_{k=1}^{K} \hat{L}_{k} U\left(z_{1}, \ldots, z_{K}\right)=B \prod_{k=1}^{K}\left(\frac{\partial U}{\partial z_{k}}\right)^{r_{k}}
$$

Here $r_{k}=\sum_{n \in I_{k}} \beta_{n}, B=b \prod_{n=1}^{N} c_{n}^{\beta_{n}}$. The linear differential operator $\hat{L}_{k}$ of order $M_{k}$ acting in the variable $z_{k}$ is of the form

$$
\hat{L}_{k}=\sum_{m=1}^{M_{k}} A_{k}^{(m)} \frac{\partial^{m}}{\partial z_{k}^{m}}
$$

where the coefficients of the operator are $A_{k}^{(m)}=\sum_{\sigma_{k}^{(m)}} a_{\sigma_{k}^{(m)}} \prod_{n \in I_{k}} c_{n}^{m_{n}}$.

Thus, original equation (1) is transformed to the reduced equation (6) for the solutions depending on the variables $z_{k}$ and determined by expression (5). 


\section{Auxiliary FUnCtional Differential EQUATION}

For further analysis of the solutions to equations (6) we consider an auxiliary functional differential equation (FDE) for unknown functions $U_{k}\left(z_{k}\right)(k=1, \ldots, K)$ :

$$
\sum_{k=1}^{K} \hat{P}_{k} U_{k}\left(z_{k}\right)=B \prod_{k=1}^{K} \hat{N}_{k} U_{k}\left(z_{k}\right),
$$

where $\hat{P}_{k}, \hat{N}_{k}$ are differential operators in the variable $z_{k}$.

Lemma 1. Equation (8) is satisfied by the functions $U_{k}\left(z_{k}\right)$, which are solutions of the following ordinary differential equations:

1) Under one of the conditions $B=0$ or $\hat{N}_{l} U_{l}\left(z_{l}\right) \equiv 0$ for some $l \in \Omega$,

$$
\hat{P}_{k} U_{k}\left(z_{k}\right)=\mu_{k},
$$

for all $k \in \Omega$, here the constants $\mu_{k}$ should obey the condition

$$
\sum_{k=1}^{K} \mu_{k}=0
$$

2) As $B \neq 0$, for each fixed $l \in \Omega$,

$$
\hat{P}_{l} U_{l}\left(z_{l}\right)+\tilde{\mu}_{l}=B \tilde{\nu}_{l} \hat{N}_{l} U_{l}\left(z_{l}\right),
$$

and for all $k \in \Omega, k \neq l$, the functions $U_{k}\left(z_{k}\right)$ solve the systems

$$
\hat{P}_{k} U_{k}\left(z_{k}\right)=\mu_{k}, \quad \hat{N}_{k} U_{k}\left(z_{k}\right)=\nu_{k} .
$$

Here

$$
\tilde{\mu}_{l}=\sum_{k=1, k \neq l}^{K} \mu_{k}, \quad \tilde{\nu}_{l}=\prod_{k=1, k \neq l}^{K} \nu_{k},
$$

$\mu_{k}, \nu_{k}$ are some constants $\left(\nu_{k} \neq 0\right)$. In particular, equation (8) is satisfied by the functions $U_{k}\left(z_{k}\right)$ solving equations(12) for all $k \in \Omega$ if the constants $\mu_{k}, \nu_{k}$ obey the condition

$$
\sum_{k=1}^{K} \mu_{k}=B \prod_{k=1}^{K} \nu_{k}
$$

Proof. 1. If one of the conditions $B=0$ or $\hat{N}_{l} U_{l}\left(z_{l}\right) \equiv 0$ for some $l \in \Omega$ is satisfied, then equation (8) is reduced to the following one:

$$
\sum_{k=1}^{K} \hat{P}_{k} U_{k}\left(z_{k}\right)=0 .
$$

Since the left hand side of equation (15) is a sum of functions of different variables $z_{k}$, then the functions $U_{k}\left(z_{k}\right)$ should satisfy equation (9), while the constants $\mu_{k}$ should obey condition (10).

2. Assume that the right hand side in (8) is not identically zero and consider the case, when all the factors in the right hand side of (8) are non-zero constants. In this case the functions $U_{k}\left(z_{k}\right)$ satisfy the second of equations (12), for all $k \in \Omega$ we have $\nu_{k} \neq 0$, and equation (8) is reduced to the following one:

$$
\sum_{k=1}^{K} \hat{P}_{k} U_{k}\left(z_{k}\right)=B \prod_{k=1}^{K} \nu_{k}
$$

Arguing for equation (16) as above, we obtain that the functions $U_{k}\left(z_{k}\right)$ should satisfy the first of equations (12), while the constants $\mu_{k}, \nu_{k}$ should obey condition (14). At that, equation (8) 
is satisfied if and only if systems (12) are compatible for all $k \in \Omega$. Let $l \in \Omega$ be some fixed value $k$, for which the condition

$$
\hat{N}_{l} U_{l}\left(z_{l}\right) \neq \text { const }
$$

holds. We differentiate equation (8) term by term w.r.t. $z_{l}$ and in view of (17), we write it as

$$
\frac{\left(\partial / \partial z_{l}\right) \hat{P}_{l} U_{l}\left(z_{l}\right)}{\left(\partial / \partial z_{l}\right) \hat{N}_{l} U_{l}\left(z_{l}\right)}=B \prod_{k=1, k \neq l}^{K} \hat{N}_{k} U_{k}\left(z_{k}\right) .
$$

The left hand side of relation (18) depends only on $z_{l}$, while the right hand side depends only on $z_{k}, k \neq l$. Hence, it is satisfied only in the case, when all functions $U_{k}\left(z_{k}\right)$ solve the second equation in (12) for all $k \neq l$. Then equation (8) can be reduced to the form:

$$
\sum_{k=1}^{K} \hat{P}_{k} U_{k}\left(z_{k}\right)=B \tilde{\nu}_{l} \hat{N}_{l} U_{l}\left(z_{l}\right),
$$

where $\tilde{\nu}_{l}$ is determined by the second identity in (13). Since the right hand side in (19) depends only on $z_{l}$, this equation can be satisfied only in the case, when the left hand side depends only on this variable. This implies that for all $k \neq l$ the functions $U_{k}\left(z_{k}\right)$ should satisfy the first equation in (12). Then equation (8) is satisfied if $U_{l}\left(z_{l}\right)$ solves equation (11), where the constants $\tilde{\nu}_{l}, \tilde{\mu}_{l}$ are determined by expressions (13). Thus, for each $l \in \Omega$, for which condition (17) is satisfied, the function $U_{l}\left(z_{l}\right)$ is determined by solving equation (11) and the functions $U_{k}\left(z_{k}\right)$ for $k \neq l$ are solutions to system (12). The considered solution exists if and only if these systems are compatible for all $k \neq l$. The proof is complete.

\section{ANALYSis OF REDUCED EQUATION}

In this section we analyse the solutions to equation (6). First we consider simplest particular cases.

I. The right hand side in (6) is identically zero.

1) $B=0$. In this case (6) is reduced to the linear homogeneous equation:

$$
\sum_{k=1}^{K} \hat{L}_{k} U\left(z_{1}, \ldots, z_{K}\right)=0
$$

In particular, if the parameters of the problem are such that together with the condition $B=0$, the conditions $A_{k}^{(m)}=0$ are satisfied for all $k \in \Omega, 1 \leqslant m \leqslant M_{k}$, then equation (20), and therefore, equation (6) holds for an arbitrary function $U\left(z_{1}, \ldots, z_{K}\right)$ differentiable sufficiently many times in all variables.

2) If for some $l \in \Omega$ the condition $r_{l}>0$ holds, then equation (6) is satisfied by each solution to the following linear homogeneous equation

$$
\sum_{k=1, k \neq l}^{K} \hat{L}_{k} U\left(z_{1}, \ldots, z_{l-1}, z_{l+1}, \ldots, z_{K}\right)=0 .
$$

Similar to Case 1), if for all $k \in \Omega, k \neq l, 1 \leqslant m \leqslant M_{k}$ the conditions $A_{k}^{(m)}=$ 0 hold, then equation (21), and therefore, equation (6), holds for an arbitrary function $U\left(z_{1}, \ldots, z_{l-1}, z_{l+1}, \ldots, z_{K}\right)$ differentiable sufficiently many times in all variables.

II. General case. 
Theorem 1. (On additive separation of variables). Equation (6) has the following family of solutions represented by the sum of the functions of one of the variables $z_{1}, \ldots, z_{K}$ :

$$
U\left(z_{1}, \ldots, z_{K}\right)=U_{l}\left(z_{l}\right)+\sum_{k=1, k \neq l}^{K} \nu_{k}^{1 / r_{k}} z_{k}+U_{0} .
$$

At that, the function $U_{l}\left(z_{l}\right)$ solves the following ordinary differential equation:

$$
\hat{L}_{l} U_{l}\left(z_{l}\right)+\tilde{\mu}_{l}=B \tilde{\nu}_{l}\left[U_{l}^{\prime}\left(z_{l}\right)\right]^{r_{l}} .
$$

Here $U_{0}, \nu_{k}$ are arbitrary constants; $\tilde{\mu}_{l}, \tilde{\nu}_{l}$ are determined by the expressions:

$$
\tilde{\nu}_{l}=\prod_{k=1, k \neq l}^{K} \nu_{k}, \quad \tilde{\mu}_{l}=\sum_{k=1, k \neq l}^{K} \nu_{k}^{1 / r_{k}} A_{k}^{(1)} .
$$

Hereinafter the operator $\hat{L}_{l}$ is determined by the expression (7). Solution (22) exists for all $l \in \Omega$.

Proof. According to the known scheme of the additive separation of variables [2], we seek the solution to equation (6) as

$$
U\left(z_{1}, \ldots, z_{K}\right)=\sum_{k=1}^{K} U_{k}\left(z_{k}\right) .
$$

Substituting (25) into equation (6), we obtain the following:

$$
\sum_{k=1}^{K} \hat{L}_{k} U_{k}\left(z_{k}\right)=B \prod_{k=1}^{K}\left[U_{k}^{\prime}\left(z_{k}\right)\right]^{r_{k}} .
$$

Relation (26) is a FDE of form (8); at that,

$$
\hat{N}_{k} U_{k}\left(z_{k}\right)=\left[U_{k}^{\prime}\left(z_{k}\right)\right]^{r_{k}} .
$$

In accordance with the said above, in the cases, when the right hand side of equation (6) is identically zero, it is reduced to linear equations (20) or (21). This is why we assume that the right hand side in (6) is not identically zero.

Assume we are given some $l \in \Omega$. Then by Lemma 1, equation (26) is satisfied by the function $U_{l}\left(z_{l}\right)$ solving equation (23) and by the functions $U_{k}\left(z_{k}\right), k \neq l$, solving the following systems:

$$
\hat{L}_{k} U_{k}\left(z_{k}\right)=\mu_{k}, \quad\left[U_{k}^{\prime}\left(z_{k}\right)\right]^{r_{k}}=\nu_{k} .
$$

Solving (28) and taking into consideration expression (7), we find:

$$
U_{k}\left(z_{k}\right)=\nu_{k}^{1 / r_{k}} z_{k}+U_{k 0}
$$

where $U_{k 0}$ is an arbitrary constant. At that, system (28) is compatible if and only if the constants $\mu_{k}, \nu_{k}$ satisfy the relation

$$
\mu_{k}=\nu_{k}^{1 / r_{k}} A_{k}^{(1)}
$$

where $A_{k}^{(1)}$ is the coefficient at the first derivative in expression (7). By (30) and (13) we obtain expression (24) for the constant $\tilde{\mu}_{l}$. Then, substituting (29) into (25) and summing additive constants $U_{k 0}$, we obtain solution (22). The proof is complete.

Theorem 2. (On multiplicative separation of variables). Equation (6) has the following families of solutions represented as the product of the functions on one variables $z_{1}, \ldots, z_{K}$ : 
1) in the case $r_{\Sigma} \neq 1$ :

$$
U\left(z_{1}, \ldots, z_{K}\right)=\tilde{\nu}_{l}^{\frac{1}{r^{-1}}} U_{l}\left(z_{l}\right) \prod_{k=1, k \neq l}^{K} \rho_{k}^{-\rho_{k}}\left(z_{k}-z_{k 0}\right)^{\rho_{k}},
$$

where $\rho_{k}, r_{\Sigma}$ are determined by the expressions

$$
\rho_{k}=\frac{r_{k}}{r_{\Sigma}-1}, \quad r_{\Sigma}=\sum_{k=1}^{K} r_{k},
$$

and function $U_{l}\left(z_{l}\right)$ solve the ordinary differential equation

$$
\hat{L}_{l} U_{l}\left(z_{l}\right)=B \tilde{\nu}_{l}\left[U_{l}^{\prime}\left(z_{l}\right)\right]^{r_{l}}\left[U_{l}\left(z_{l}\right)\right]^{r_{\Sigma}-r_{l}} .
$$

Solution (31) exists if for all $k \neq l, k \in \Omega$, for each $1 \leqslant m \leqslant M_{k}$, at least one of the following conditions holds:

$$
\begin{aligned}
& A_{k}^{(m)}=0, \\
& r_{k}=\left(r_{\Sigma}-1\right) \tilde{m}_{k}
\end{aligned}
$$

for some integer $\tilde{m}_{k}$ such that $1 \leqslant \tilde{m}_{k} \leqslant m-1$.

2) in the case $r_{\Sigma}=1$ :

$$
U\left(z_{1}, \ldots, z_{K}\right)=C_{0} U_{l}\left(z_{l}\right) \exp \left(\sum_{k=1, k \neq l}^{K} \lambda_{k} z_{k}\right) .
$$

Here $C_{0}, \lambda_{k}$ are arbitrary constants and the function $U_{l}\left(z_{l}\right)$ is the solution of the ordinary differential equation

$$
\hat{L}_{l} U_{l}\left(z_{l}\right)+\tilde{\mu}_{l} U_{l}\left(z_{l}\right)=B \tilde{\nu}_{l}\left[U_{l}^{\prime}\left(z_{l}\right)\right]^{r_{l}}\left[U_{l}\left(z_{l}\right)\right]^{1-r_{l}} .
$$

The coefficients $\tilde{\mu}_{l}, \tilde{\nu}_{l}$ involved in (37) are determined by the expressions:

$$
\tilde{\nu}_{l}=\prod_{k=1, k \neq l}^{K} \lambda_{k}^{r_{k}}, \quad \tilde{\mu}_{l}=\sum_{k=1, k \neq l}^{K} \sum_{m=1}^{M_{k}} \lambda_{k}^{m} A_{k}^{(m)} .
$$

Proof. We seek a solution to equation (6) as

$$
U\left(z_{1}, \ldots, z_{K}\right)=\prod_{k=1}^{K} U_{k}\left(z_{k}\right)
$$

We substitute (39) into equation (6) and after some transformations we obtain

$$
\sum_{k=1}^{K} \frac{\hat{L}_{k} U_{k}\left(z_{k}\right)}{U_{k}\left(z_{k}\right)}=B \prod_{k=1}^{K}\left\{\left[U_{k}^{\prime}\left(z_{k}\right)\right]^{r_{k}}\left[U_{k}\left(z_{k}\right)\right]^{r_{\Sigma}-r_{k}-1}\right\} .
$$

Equation (40), as the above considered equation (26), is a FDE of form (8). The operators involved in this FDE are of the form:

$$
\hat{P}_{k} U_{k}\left(z_{k}\right)=\frac{\hat{L}_{k} U_{k}\left(z_{k}\right)}{U_{k}\left(z_{k}\right)} ; \quad \hat{N}_{k} U_{k}\left(z_{k}\right)=\left[U_{k}^{\prime}\left(z_{k}\right)\right]^{r_{k}}\left[U_{k}\left(z_{k}\right)\right]^{r_{\Sigma}-r_{k}-1} .
$$

Now we consider the cases listed in the formulation of the theorem.

1. Case $r_{\Sigma} \neq 1$. If $l \in \Omega$ is some fixed value of the index $k$, by the second equation in system (12) we find that for all $k \neq l, U_{k}\left(z_{k}\right)$ is determined by the expression:

$$
U_{k}\left(z_{k}\right)=U_{k 0}\left(z_{k}-z_{k 0}\right)^{\rho_{k}}, \quad U_{k 0}=\left(\frac{\lambda_{k}}{\rho_{k}}\right)^{\rho_{k}},
$$


where $\rho_{k}$ is determined by the expression (32), $\lambda_{k}=\nu_{k}^{1 / r_{k}}$. We substitute (42) into the first equation in system (12) and take into consideration (41). Then after some elementary transformations we obtain:

$$
\sum_{m=1}^{M_{k}} Q_{k}^{(m)}\left(\rho_{k}\right) A_{k}^{(m)}\left(z_{k}-z_{k 0}\right)^{\rho_{k}-m}=\mu_{k}\left(z_{k}-z_{k 0}\right)^{\rho_{k}},
$$

where

$$
Q_{k}^{(m)}\left(\rho_{k}\right)=\rho_{k}\left(\rho_{k}-1\right) \ldots\left(\rho_{k}-m+1\right) .
$$

Equation (43) can be satisfied only if the conditions

$$
Q_{k}^{(m)}\left(\rho_{k}\right) A_{k}^{(m)}=0, \quad \mu_{k}=0
$$

hold for each $k \neq l, k \in \Omega$ and all $m=1, \ldots, M_{k}$. In view of expression (44), it is obvious that for each given $m$ the first of conditions (45) is satisfied if either $A_{k}^{(m)}=0$ or $\rho_{k}=\tilde{m}_{k}$ for some $1 \leqslant \tilde{m}_{k} \leqslant m-1$. This implies that the considered solution exists if and only if at least one of conditions (34), (35) holds true. Employing Lemma 1 and equation (11), in view of (13) and the second condition in (45), we arrive at equation (33) for the function $U_{l}\left(z_{l}\right)$. Substituting expression (42) into (39), we obtain the solution of form (31).

2. Case $r_{\Sigma}=1$. In this case the second equation in system (12) is of the form

$$
\left(\frac{U_{k}^{\prime}\left(z_{k}\right)}{U_{k}\left(z_{k}\right)}\right)^{r_{k}}=\nu_{k}
$$

and this implies that for all $k \neq l$

$$
U_{k}\left(z_{k}\right)=U_{k 0} \exp \left(\lambda_{k} z_{k}\right),
$$

where $\lambda_{k}=\nu_{k}^{1 / r_{k}}$ as in the previous case.

Substituting (46) into the first equation of system (12), in view of the first relation in (41) we obtain:

$$
\sum_{m=1}^{M_{k}} A_{k}^{(m)} \lambda_{k}^{m}=\mu_{k}
$$

Then due to (47) and (13) by equation (11) we find that the function $U_{l}\left(z_{l}\right)$ should solve equation (37), in which $\tilde{\nu}_{l}, \tilde{\mu}_{l}$ are determined by expressions (38). Substituting expression (46) into (39), we obtain the solution in form (36). The proof is complete.

Let the set $\Omega$ be represented as the union $S$ of disjoint subsets $\Omega_{s}, s=1, \ldots, S$. In what follows we employ the expression:

$$
r_{\Sigma s}=\sum_{k \in \Omega_{s}} r_{k}
$$

We also introduce $\Lambda_{0}$ as the set of the values of the index $s$, for which $r_{\Sigma s}=0$. Hereafter we assume that $l, t$ are some fixed values of the indices $k, s$ and $l \in \Omega_{t}$.

Theorem 3. (on combined separation of variables) For each partition of the set $\Omega$ into the subsets $\Omega_{s}, s=1, \ldots, S$, equation (6) has the following family of solutions:

a) as $r_{\Sigma t}=1$ :

$$
\begin{aligned}
U\left(z_{1}, \ldots, z_{K}\right)= & \sum_{s \in \Lambda_{0}, s \neq t} D_{s} \exp \left(\sum_{k \in \Omega_{s}} \lambda_{k} z_{k}\right) \\
& +\sum_{s \notin \Lambda_{0}, s \neq t} E_{s} \prod_{k \in \Omega_{s}}\left(z_{k}-z_{k 0}\right)^{\sigma_{k}}+D_{t} \exp \left(\sum_{k \in \Omega_{t}, k \neq l} \lambda_{k} z_{k}\right) U_{l}\left(z_{l}\right) .
\end{aligned}
$$


At that, $U_{l}\left(z_{l}\right)$ solves the ordinary differential equation:

$$
\hat{L}_{l} U_{l}\left(z_{l}\right)+\mu_{l} U_{l}\left(z_{l}\right)=F_{l}\left[U_{l}^{\prime}\left(z_{l}\right)\right]^{r_{l}}\left[U_{l}\left(z_{l}\right)\right]^{1-r_{l}},
$$

where

$$
\mu_{l}=\sum_{k \in \Omega_{t}, k \neq l} \sum_{m=1}^{M_{k}} A_{k}^{(m)} \lambda_{k}^{m}, \quad F_{l}=B \prod_{k \in \Omega_{t}, k \neq l}^{K} \lambda_{k}^{r_{k}} \prod_{s \in \Lambda_{0}, s \neq t} \prod_{k \in \Omega_{s}} \lambda_{k}^{r_{k}} \prod_{s \notin \Lambda_{0}, s \neq t} \prod_{k \in \Omega_{s}} \sigma_{k}^{r_{k}} \prod_{s \notin \Lambda_{0}, s \neq t} E_{s}^{r_{\Sigma s}} .
$$

Solution (48) exists under the following conditions:

$$
\sum_{k \in \Omega_{s}} \sum_{m=1}^{M_{k}} A_{k}^{(m)} \lambda_{k}^{m}=0
$$

for all $s \in \Lambda_{0}, s \neq t$

$$
A_{k}^{(m)} Q_{k}^{(m)}\left(\sigma_{k}\right)=0
$$

for all $k \in \Omega_{s}, 1 \leqslant m \leqslant M_{k}, s \notin \Lambda_{0}, s \neq t$;

b) as $r_{\Sigma t} \neq 1$ :

$$
\begin{aligned}
U\left(z_{1}, \ldots, z_{K}\right)= & \sum_{s \in \Lambda_{0}, s \neq t} D_{s} \exp \left(\sum_{k \in \Omega_{s}} \lambda_{k} z_{k}\right) \\
& +\sum_{s \notin \Lambda_{0}, s \neq t} E_{s} \prod_{k \in \Omega_{s}}\left(z_{k}-z_{k 0}\right)^{\sigma_{k}}+E_{t} U_{l}\left(z_{l}\right) \prod_{k \in \Omega_{t}, k \neq l}\left(z_{k}-z_{k 0}\right)^{\rho_{k}} .
\end{aligned}
$$

At that, $U_{l}\left(z_{l}\right)$ solves the ordinary differential equation:

$$
\hat{L}_{l} U_{l}\left(z_{l}\right)=G_{l}\left[U_{l}^{\prime}\left(z_{l}\right)\right]^{r_{l}}\left[U_{l}\left(z_{l}\right)\right]^{r_{\Sigma t}-r_{l}}
$$

where

$$
G_{l}=B E_{t}^{r_{\Sigma t}-1} \prod_{k \in \Omega_{t}, k \neq l}^{K} \rho_{k}^{r_{k}} \prod_{s \in \Lambda_{0}, s \neq t} \prod_{k \in \Omega_{s}} \lambda_{k}^{r_{k}} \prod_{s \notin \Lambda_{0}, s \neq t} \prod_{k \in \Omega_{s}} \sigma_{k}^{r_{k}} \prod_{s \notin \Lambda_{0}, s \neq t} E_{s}^{r_{\Sigma s}} .
$$

Solution (52) exists under conditions (50), (51) and the following additional condition:

$$
A_{k}^{(m)} Q_{k}^{(m)}\left(\rho_{k}\right)=0
$$

for all $k \in \Omega_{t}, k \neq l, 1 \leqslant m \leqslant M_{k}$. In formulae (48)-(54), the symbols $D_{s}, E_{s}, \lambda_{k}, z_{k 0}$ stand for arbitrary constants, while $\rho_{k}, \sigma_{k}$ are determined by the expressions:

$$
\begin{aligned}
\rho_{k} & =\frac{r_{k}}{r_{\Sigma t}-1} \quad\left(k \in \Omega_{t}\right), \\
\sigma_{k} & =\frac{r_{k}}{r_{\Sigma s}} \quad\left(k \in \Omega_{s}\right) .
\end{aligned}
$$

Proof. We seek solutions to equation (6) as

$$
U\left(z_{1}, \ldots, z_{K}\right)=\sum_{s=1}^{S} \prod_{k \in \Omega_{s}} U_{k}\left(z_{k}\right)
$$

Substituting (57) into equation (6) and taking into consideration expression (7), we reduce equation (6) to the form:

$$
\sum_{s=1}^{S} \sum_{k \in \Omega_{s}} \hat{P}_{k} U_{k}\left(z_{k}\right) \prod_{k \in \Omega_{s}} U_{k}\left(z_{k}\right)=B \prod_{s=1}^{S} \prod_{k \in \Omega_{s}}\left\{\left[U_{k}^{\prime}\left(z_{k}\right)\right]^{r_{k}}\left[U_{k}\left(z_{k}\right)\right]^{r_{\Sigma s}-r_{k}}\right\}
$$


where $\hat{P}_{k}\left[U_{k}\left(z_{k}\right)\right]$ is determined by the first identity in (41). We differentiate equation (58) term by term in $z_{l}$ to obtain:

$$
\frac{\partial}{\partial z_{l}}\left\{\sum_{k \in \Omega_{t}} \hat{P}_{k} U_{k}\left(z_{k}\right) \prod_{k \in \Omega_{t}} U_{k}\left(z_{k}\right)\right\}=B \prod_{k \in \Omega, k \neq l}\left\{\left[U_{k}^{\prime}\left(z_{k}\right)\right]^{r_{k}}\left[U_{k}\left(z_{k}\right)\right]^{r_{\Sigma s}-r_{k}}\right\} \frac{\partial}{\partial z_{l}}\left\{\left[U_{l}^{\prime}\left(z_{l}\right)\right]^{r_{l}}\left[U_{l}\left(z_{l}\right)\right]^{r_{\Sigma t}-r_{l}}\right\} .
$$

The left hand side in (59) depends only on $z_{k}\left(k \in \Omega_{t}\right)$ and this is why for all $k \in \Omega_{s}, s \neq t$ the functions $U_{k}\left(z_{k}\right)$ should satisfy the equation:

$$
\left[U_{k}^{\prime}\left(z_{k}\right)\right]^{r_{k}}\left[U_{k}\left(z_{k}\right)\right]^{r_{\Sigma s}-r_{k}}=\lambda_{k}^{r_{k}}
$$

where $\lambda_{k}$ are some constants. We consider particular cases for equation (60).

1) $r_{\Sigma s}=0$. In this case, the solution to equation (60) is the exponential function:

$$
\left.U_{k}\left(z_{k}\right)\right]=U_{k 0} \exp \left(\lambda_{k} z_{k}\right)
$$

2) $r_{\Sigma s} \neq 0$. In this case the solution to equation (60) is of the form:

$$
\left.U_{k}\left(z_{k}\right)\right]=\left(\frac{\lambda_{k}}{\sigma_{k}}\right)^{\sigma_{k}}\left(z_{k}-z_{k 0}\right)^{\sigma_{k}}
$$

where $\sigma_{k}$ is determined by expression (56). Employing expressions (61), (62), we can write the terms in the left hand side of equation (58) corresponding to particular values $s \neq t$ as:

1) For $r_{\Sigma s}=0$ :

$$
\sum_{k \in \Omega_{s}} \hat{P}_{k}\left[U_{k}\left(z_{k}\right)\right] \prod_{k \in \Omega_{s}} U_{k}\left(z_{k}\right)=D_{s} \exp \left(\sum_{k \in \Omega_{s}} \lambda_{k} z_{k}\right) \sum_{k \in \Omega_{s}} \sum_{m=1}^{M_{k}} A_{k}^{(m)} \lambda_{k}^{m}
$$

where

$$
D_{s}=\prod_{k \in \Omega_{s}} U_{k 0}
$$

2) For $r_{\Sigma s} \neq 0$ :

$$
\sum_{k \in \Omega_{s}} \hat{P}_{k}\left[U_{k}\left(z_{k}\right)\right] \prod_{k \in \Omega_{s}} U_{k}\left(z_{k}\right)=E_{s} \prod_{k \in \Omega_{s}}\left(z_{k}-z_{k 0}\right)^{\sigma_{k}} \sum_{k \in \Omega_{s}} \sum_{m=1}^{M_{k}} A_{k}^{(m)} Q_{k}^{(m)}\left(\sigma_{k}\right)\left(z_{k}-z_{k 0}\right)^{-m},
$$

where $Q_{k}^{(m)}$ is determined by expression (44),

$$
E_{s}=\prod_{k \in \Omega_{s}}\left(\frac{\lambda_{k}}{\sigma_{k}}\right)^{\sigma_{k}}
$$

As it was mentioned above, the functions $U_{k}\left(z_{k}\right)$ satisfy equation (60) for all $k \in \Omega_{s}, s \neq t$. This is why both the right hand side and left hand side of equation (58) can depend only of the variables $z_{k}, k \in \Omega_{t}$. In view of expressions (63), (64), this is possible only under conditions (50), (51). Then equation (58) becomes:

$$
\sum_{k \in \Omega_{t}} \hat{P}_{k} U_{k}\left(z_{k}\right)=\tilde{B}_{t} \prod_{k \in \Omega_{t}}\left\{\left[U_{k}^{\prime}\left(z_{k}\right)\right]^{r_{k}}\left[U_{k}\left(z_{k}\right)\right]^{r_{\Sigma t}-r_{k}-1}\right\}
$$

where

$$
\tilde{B}_{t}=B \prod_{s=1, s \neq t}^{S} \prod_{k \in \Omega_{s}} \lambda_{k}^{r_{k}}
$$


Arguing as in the proof of (59), we differentiate equation (65) term by term w.r.t. $z_{l}$ and as a result we obtain

$$
\frac{\partial}{\partial z_{l}}\left\{\hat{P}_{l} U_{l}\left(z_{l}\right)\right\}=\tilde{B}_{t} \prod_{k \in \Omega_{t}, k \neq l}\left\{\left[U_{k}^{\prime}\left(z_{k}\right)\right]^{r_{k}}\left[U_{k}\left(z_{k}\right)\right]^{r_{\Sigma s}-r_{k}-1}\right\} \frac{\partial}{\partial z_{l}}\left\{\left[U_{l}^{\prime}\left(z_{l}\right)\right]^{r_{l}}\left[U_{l}\left(z_{l}\right)\right]^{r_{\Sigma t}-r_{l}-1}\right\} .
$$

Since the left hand side of equation (66) depends only on $z_{l}$, the right hand side can depend only on this variable and this is why the functions $U_{k}\left(z_{k}\right)$ should satisfy the equation

$$
\left[U_{k}^{\prime}\left(z_{k}\right)\right]^{r_{k}}\left[U_{k}\left(z_{k}\right)\right]^{r_{\Sigma t}-r_{k}-1}=\lambda_{k}^{r_{k}}
$$

for all $k \in \Omega_{t}, k \neq l$.

Equation (67) has the form similar to equation (60) up to the change $r_{\Sigma s} \rightarrow r_{\Sigma t}-1$ and this is why, as in the analysis of equation (60), we consider the particular cases:

1) $r_{\Sigma t}=1$. In this case function (61) solves equation (67). Substituting expression (61) into equation (65), we obtain that the function $U_{l}\left(z_{l}\right)$ should satisfy equation (49). Employing expressions (61), (62), (57), we obtain equation in the form (48).

2) $r_{\Sigma t} \neq 1$. In this case equation (67) is of the form:

$$
U_{k}\left(z_{k}\right)=\left(\frac{\lambda_{k}}{\rho_{k}}\right)^{\rho_{k}}\left(z_{k}-z_{k 0}\right)^{\rho_{k}}
$$

where $\rho_{k}$ is determined by expression (55).

Substituting expression (68) into equation (65), we obtain:

$$
\hat{P}_{l} U_{l}\left(z_{l}\right)+\sum_{k \in \Omega_{t}, k \neq l} \sum_{m=1}^{M_{k}} A_{k}^{(m)} Q_{k}^{(m)}\left(\rho_{k}\right)\left(z_{k}-z_{k 0}\right)^{-m}=G_{l}\left[U_{l}^{\prime}\left(z_{l}\right)\right]^{r_{l}}\left[U_{l}\left(z_{l}\right)\right]^{r_{\Sigma t}-r_{l}-1} .
$$

If condition (54) is satisfied for all $k \in \Omega_{t}, k \neq l, 1 \leqslant m \leqslant M_{k}$, then equation (69) is reduced to ordinary differential equation (53) for the function $U_{l}\left(z_{l}\right)$. Substituting expressions (61), (62), (68) into (57), we obtain the solution to equation (6) in the form (52). The proof is complete.

\section{Conclusion}

Thus, in the present work we studied multidimensional partial differential equation (1) involving linear differential operator of arbitrary order and power nonlinearities in the first derivatives. For solutions of multi-dimensional travelling waves type depending on some linear combinations of original variables, equation (1) is transformed to the reduced equation (6). In order to solve this equation, we apply the separation of variables. At that we first analyse the auxiliary functional differential equation arising while applying this method to the reduced equation. We obtain solutions of the reduced equations for additive, multiplicative and combined separation of variables.

\section{BIBLIOGRAPHY}

1. A.D. Polyanin, V.F. Zaitsev. Handbook on nonlinear equations of mathematics physics: exact solutions. Fizmatlit, Moscow (2002). (in Russian).

2. A.D. Polyanin, V.F. Zaitsev, A.I. Zhurov. Methods for solving nonlinear equations of mathematical physics and mechanics. Fizmatlit, Moscow (2005).

3. V.F. Zaitsev, A.D. Polyanin. Handbook of first order partial differential equations. Fizmatlit, Moscow (2002). [A.D. Polyanin, V.F. Zaitsev, A. Moussiaux. Taylor \& Francis, London (2002).]

4. A.D. Polyanin, A.I. Zhurov. The generalized and functional separation of variables in mathematical physics and mechanics // Dokl. Akad. Nauk. 382:5, 606-611 (2002). [Dokl. Math. 65:1, $129-134$ (2002).] 
5. I.V. Rakhmelevich. On two-dimensional hyperbolic equations with power-law non-linearity in the derivatives // Vestn. Tomsk. Gos. Univ. Matem. Mekh. 1, 12-19 (2015). (in Russian).

6. I.V. Rakhmelevich. On some new solutions of the multi-dimensional first order partial differential equation with power-law non-linearities // Vestn. Tomsk. Gos. Univ. Matem. Mekh. 3, 18-25 (2015). (in Russian).

7. I.V. Rakhmelevich. On application of the variable separation method to mathematical physics equations containing homogeneous functions of derivatives // Vestn. Tomsk. Gos. Univ. Matem. Mekh. 3, 37-44 (2013). (in Russian).

8. I.V. Rakhmelevich. On equations of mathematical physics containing multi-homogeneous functions of derivatives // Vestn. Tomsk. Gos. Univ. Matem. Mekh. 1, 42-50 (2013). (in Russian).

9. I.V. Rakhmelevich. On the Solutions of Multi-dimensional Clairaut Equation with Multihomogeneous Function of the Derivatives // Izv. Saratov Univ. N.S. Ser. Math. Mech. Inform. 14:4(1), 374-381 (2014).

10. J. Miller (Jr.), Rubel L.A. Functional separation of variables for Laplace equations in two dimensions // J. Phys. A. 26:8, 1901-1913 (1993).

11. R.Z. Zhdanov. Separation of variables in the non-linear wave equation // J. Phys. A. 27:9, L291L297 (1994).

12. A.M. Grundland, E. Infeld. A family of non-linear Klein-Gordon equations and their solutions // J. Math. Phys. 33:7, 2498-2503 (1992).

Igor Vladimirovich Rakhmelevich, Lobachevsky State University of Nizhni Novgorod, Gagarin av. 23, 603950, Nizhni Novgorod, Russia

E-mail: igor-kitpd@yandex.ru 